HEART FAILURE

\title{
Interarterial shunt device for HFpEF
}

A novel interarterial shunt device (IASD) provides sustained clinical benefit and is safe for patients with heart failure and preserved ejection fraction (HFpEF) 1 year after implantation. These 1-year outcomes were presented at the AHA Scientific Sessions 2016 and published in Circ. Heart Fail. REDUCE LAP-HF is a multicentre, prospective, nonrandomized, open-label, single-arm study designed to assess the performance and safety of an IASD in patients with symptomatic HFpEF. In total, 64 patients with left ventricular ejection fraction $\geq 40 \%$, NYHA class II-IV, and elevated pulmonary capillary wedge pressure ( $\geq 15 \mathrm{mmHg}$ at rest or $\geq 25 \mathrm{mmHg}$ during exercise) were implanted with an IASD. At 12 months, improvements in quality-of-life score $(P<0.001)$, NYHA class $(P<0.001)$, and 6 -min walking distance $(P<0.01)$ were observed. Modest, but stable, reductions in left ventricular end-diastolic volume index were observed on echocardiography, together with an increase in right ventricular end-diastolic volume index.

Given the concern that continually increased right-sided blood flow could negatively influence pulmonary pressures, a subset of patients
( $n=18)$ underwent catheterization for rest and exercise haemodynamic testing. These patients showed increased exercise time from baseline to 6 months ( $8.2 \mathrm{~min}$ vs $9.7 \mathrm{~min} ; P<0.05$ ), and this increase was maintained at 12 months (10.4 min; $P<0.05$ vs baseline). Furthermore, supine exercise peak work capacity was also increased at 6 months compared with baseline, and maintained at 12 months. This increase in work capacity did not result in an increase in pulmonary capillary wedge pressure. No evidence of adverse remodelling after IASD placement was detected.

"This study provides the longest experience with an [IASD] specifically developed for the management of HFpEF," conclude the investigators. Randomized trials involving patient and physician blinding are currently ongoing to confirm these findings.

Karina Huynh

ORIGINAL ARTICLE Kaye, D. M. et al. One year outcomes after transcatheter insertion of an interatrial shunt device for the management of heart failure with preserved ejection fraction. Circ. Heart Fail. http://dx.doi.org/10.1161/ CIRCHEARTFAILURE.116.003662 (2016) 